\title{
Note on sparsity in signal recovery and in matrix identification
}

\author{
Götz E. Pfander*
}

School of Engineering and Science, Jacobs University Bremen, 28759 Bremen, Germany.

We describe a connection between the identification problem for matrices with sparse representations in given matrix dictionaries and the problem of sparse signal recovery. This allows the application of novel compressed sensing techniques to operator identification problems such as the channel measurement problem in communications engineering.

Keywords. Sparse signal recovery, compressed sensing, Basis Pursuit, time-frequency shifts.

The goal in sparse signal recovery is the reconstruction of signals in $n$-dimensional real or complex space from $m$ measurements, $m<n$, on the basis that the signal has a representation involving only $k \leq m$ elements from a given dictionary (see $[2,1]$ and references within). In detail, we let $\mathcal{D}=\left\{g_{r}\right\}_{r=1, \ldots, N} \subseteq \mathbb{C}^{n}$ be a dictionary of $N$ vectors in $\mathbb{C}^{n}$. For all $k \leq m, n, N$, set

$$
\Sigma_{k}^{\mathcal{D}}=\left\{f=\sum_{r=1}^{N} \alpha_{r} g_{r} \in \mathbb{C}^{n}: \text { with at most } k \text { coefficients } \alpha_{r} \text { being nonzero }\right\} \subseteq \mathbb{C}^{n} .
$$

The standard approach to recover a signal with a sparse representation in $\mathcal{D}$ is to design a measurement matrix $\Phi_{\mathcal{D}} \in \mathbb{C}^{m \times n}$ which allows the recovery of any $f \in \Sigma_{k}^{\mathcal{D}}$ from the measurement vector $\Phi_{\mathcal{D}} f$ whenever $k$ is sufficiently small. While a minimal requirement for the recovery of any $f \in \Sigma_{k}^{\mathcal{D}}$ is that the constructed map $\Phi_{\mathcal{D}}: \Sigma_{k}^{\mathcal{D}} \longrightarrow \mathbb{C}^{m}, f \mapsto \Phi_{\mathcal{D}} f$ is injective, the recent literature on sparse signal recovery focuses mainly on numerically robust recovery methods whose application require that $\Phi_{\mathcal{D}}$ is not only injective but well conditioned on its domain.

Note that the Euclidean basis $\mathcal{E}=\left\{e_{j}\right\}$ is commonly used as a dictionary. Then $N=n$ and $f \in \Sigma_{k}^{\mathcal{D}}$ if $f$ has at most $k$ nonzero entries.

The identification of operators from a single input/output pair is another standard problem in applied sciences. For linear operators acting on finite dimensional spaces, we say that a class of operators, that is, matrices $\Sigma \subseteq \mathbb{C}^{m \times n}$, is identifiable if there is an $h \in \mathbb{C}^{n}$ such that the induced map $\varphi_{h}: \Sigma \longrightarrow \mathbb{C}^{m}, \quad M \mapsto M h \quad$ is injective.

Clearly, for $\Sigma=\mathbb{C}^{m \times n}$, the problem is ill posed. Following the sparse signal recovery notation used above, we let $\mathcal{M}=\left\{M_{r}\right\}_{r=1, \ldots, N} \subseteq \mathbb{C}^{m \times n}$ be a dictionary of $N$ matrices, and for $k \leq m, m n, N$, we set

$$
\Sigma_{k}^{\mathcal{M}}=\left\{M=\sum_{r=1}^{N} \alpha_{r} M_{r} \in \mathbb{C}^{m \times n}: \text { with at most } k \text { coefficients } \alpha_{r} \text { being nonzero }\right\} \subseteq \mathbb{C}^{m \times n} .
$$

The analogy of the problems described above is apparent: In sparse signal recovery, we design $\Phi_{\mathcal{D}} \in \mathbb{C}^{m \times n}$ which allows us to detect $f \in \Sigma_{k}^{\mathcal{D}}$ from the measurements $\Phi_{\mathcal{D}} f$ whenever $k$ is sufficiently small, that is,

\footnotetext{
*g.pfander@jacobs-university.de
} 


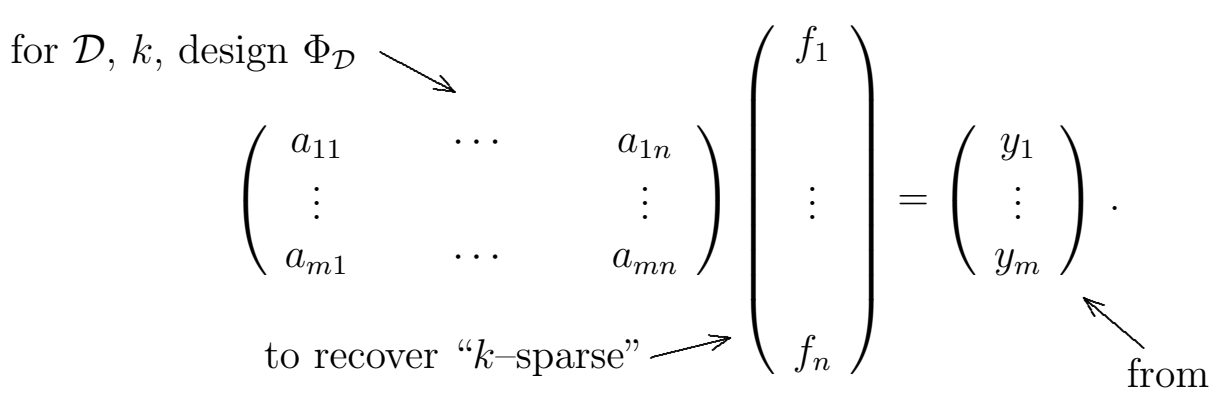

The goal in the identification problem for matrices with sparse representations on the other hand can be phrased similarly to the sparse signal recovery problem, namely by

$$
\begin{gathered}
\text { for } \mathcal{M}, k \text {, design } h \longrightarrow \\
\left.\qquad \begin{array}{ccc}
a_{11} & \ldots & a_{1 n} \\
\vdots & & \vdots \\
a_{m 1} & \ldots & a_{m n}
\end{array}\right)\left(\begin{array}{c}
h_{1} \\
\vdots \\
\text { to recover " } k \text {-sparse" } \\
h_{n}
\end{array}\right)=\left(\begin{array}{c}
y_{1} \\
\vdots \\
y_{m}
\end{array}\right) . \\
\text { from }
\end{gathered}
$$

The interconnection between sparsity in signal recovery and matrix identification is easily exposed. The recovery of $M=\sum_{r=1}^{N} \alpha_{r} M_{r} \in \Sigma_{k}^{\mathcal{M}}$ from $M h$ can be achieved by determining the coefficients $\alpha_{r}$ from

$$
M h=\left(\sum_{r=1}^{k} \alpha_{r} M_{r}\right) h=\sum_{r=1}^{k} \alpha_{r}\left(M_{r} h\right) \in \mathbb{C}^{m} .
$$

Therefore, for $\mathcal{M} h=\left\{M_{r} h\right\}_{r}$, we observe that the sparse matrix recovery problem for the matrix dictionary $\mathcal{M}$ translates into a sparse signal recovery problem in the vector dictionary $\mathcal{M} h$. Its solution can be sought using the same methods as used for sparse signal recovery, that is, a matrix $\Phi_{\mathcal{M} h} \in \mathbb{C}^{m^{\prime} \times m}$, $m^{\prime} \leq m$, for $\mathcal{M} h$ has to be designed.

The central difference between matrix identification and sparse signal recovery is the following: solving a matrix identification problem requires first the choice of a so-called identifier $h$, which then allows the design of a matrix $\Phi_{\mathcal{M} h}$. For example, for the canonical basis $\left\{E_{i j}\right\}$ of $\mathbb{C}^{m \times n}$ not even $h$ exists with $\varphi_{h}: \Sigma_{2}^{\left\{E_{i j}\right\}} \longrightarrow \mathbb{C}^{m}$ injective. Also, the more restrictive nature of matrix identification is well-illustrated by a comparison of the degrees of freedom in the corresponding design processes. If the dictionary $\mathcal{D}$ in the signal recovery problem is a basis for $\mathbb{C}^{n}$, then $N=n$ and we can choose freely the $m n$ entries of the matrix $\Phi_{\mathcal{D}}$. On the other hand, if $\mathcal{M}$ is a matrix basis for $\mathbb{C}^{m \times n}$, then $N=m n$ and our design freedom encompasses "only" $n$ parameters for $h$ and $m^{\prime} m \leq m^{2}$ parameters for $\Phi_{\mathcal{M} h}$.

We conclude that operator identification of matrices with sparse representations in matrix dictionaries can be solved using recent techniques such as Basis Pursuit and Orthogonal Matching Pursuit from the theories of compressed sensing and sparse signal recovery. This approach is expected to supply novel and powerful identification strategies in applications such as the channel measurement problem in wireless and/or mobile communications (see $[4,7]$ for background and $[3,5,6,8]$ for initial work in this direction). 


\section{REFERENCES}

[1] E.J. Candès, J. Romberg, and T. Tao. Robust uncertainty principles: exact signal reconstruction from highly incomplete frequency information. IEEE Trans. Inform. Theory, 52(2):489-509, 2006.

[2] D.L. Donoho. Compressed sensing. IEEE Trans. Inform. Theory, 52(4):1289-1306, 2006.

[3] M. Herman and T. Strohmer. High resolution radar via compressed sensing. 2007. Preprint.

[4] J. Lawrence, G.E. Pfander, and D. Walnut. Linear independence of Gabor systems in finite dimensional vector spaces. J. Fourier Anal. Appl., 11(6):715-726, 2005.

[5] G.E. Pfander and H. Rauhut. Sparsity in time-frequency representations. Preprint, 2007.

[6] G.E. Pfander, H. Rauhut, and J. Tanner. Identification of matrices having a sparse representation. Preprint, 2007.

[7] G.E. Pfander and D. Walnut. Measurement of time-variant channels. IEEE Trans. Info. Theory, 52(11):4808-4820, 2006.

[8] S. Sanyal, S.L. Kukreja, E.J. Perreault, and D.T. Westwick. Identification of linear time varying systems using basis pursuit. In Proceedings of the IEEE EMBS 2005. 